\title{
NOTES ON THE PRODUCTION OF MATTER IN THE UNIVERSE
}

\author{
V.E. KUZMICHEV, V.V. KUZMICHEV
}

PACS 98.80.Qc, 98.80.Cq,

A model of the production of ordinary and dark matter in the decay of a hypothetical antigravitating medium in the form of a condensate of spinless massive particles, which fills the Universe, is proposed. The decays of these particles into baryons, leptons, and dark matter particles are caused by some interaction with the mass scale between the electroweak interaction and the grand unification. The observed dark energy is identified with a portion of the condensate, which has not decayed up to the instant of a measurement. The decay rate of particles of the condensate is expressed through the three parameters - the coupling constant $\alpha_{X}$, the mass scale $M_{X}$, which defines the mass of an $X$-particle as a mediator of the interaction, and the energy imparted to the decay products. Under the assumption that the decay rate of particles of the condensate is of the same order of magnitude as the Hubble expansion rate, the limits of the possible values of the mass $M_{X}$ are obtained. The coupling constant $\alpha_{X} \sim \frac{1}{70}$ at $M_{X} \sim 6 \times 10^{10} \mathrm{GeV}$ can be taken as the characteristic value. The cross-sections of the reactions, in which dark matter particles can be produced, are calculated.

\section{Introduction}

According to the modern point of view (see, e.g., [1]), the very early Universe (close to the Planck era) was filled with the relativistic matter with the kinetic energy of motion of its constituent particles (temperature $T$ ), which exceeded significantly their rest mass energy, $T \gg m_{\max }$, where $m_{\max }$ is the greatest mass of all the particles. Particles may acquire the mass after the spontaneous symmetry breaking under the cooling of the expanding Universe. When the temperature falls to the value $T \ll m_{\min }$, where $m_{\min }$ is the smallest mass of particles, matter in the Universe is found to be composed of two components, radiation and massive particles. In the early Universe, the relativistic matter itself may arise as a result of the transition of the vacuum energy of some primordial scalar field into the energy of small quantum oscillations near the equilibrium state corresponding to true or false vacuum.

When applying this model to the study of the evolution of matter in the Universe, it is convenient to rep- resent the relativistic matter in the form of a sum of two components, one of which is composed of massless particles, while another one consists of massive particles. Massless particles form the radiation, with which the reference frame can be associated [2]. In the era $T \ll m_{\min }$, the massive particles produce ordinary and dark matter observed in the Universe.

In our approach [2], the massive component in the Universe is the condensate of scalar field quanta $(\phi$ particles). Their mass $m_{\phi}$ can be expressed through the curvature of the potential energy density of a primordial scalar field. Stationary states of the condensate are characterized by a mass (energy), $M_{k}=m_{\phi}\left(k+\frac{1}{2}\right)$, where $M_{k}$ is the eigenvalue of the operator of mass-energy of a scalar field in a comoving volume, $k$ is the number of $\phi$-particles which are bosons. The condensate is a chargeless medium (for all types of charges). Therefore, a $\phi$-particle coincides with its antiparticle, $\phi=\bar{\phi}$. The momentum of a $\phi$-particle vanishes, and its spin is $s_{\phi}=0$. The direct calculations show that, for states of the Universe with the condensate mass, which exceeds significantly the Planck mass, the condensate acquires the properties of an antigravitating medium with the vacuum-type equation of state.

In this paper, we study the model of production of ordinary and dark matter in the decay of a antigravitating medium in the form of a condensate of spinless massive particles. The decays of these particles into baryons, leptons, and dark matter particles are caused by some interaction with the mass scale between the electroweak interaction and the grand unification.

\section{Matter Production}

Let us suppose that baryons $(n, p)$, leptons $\left(e^{-}, \nu_{e}\right)$, dark matter particles $(\chi)$, and their antiparticles $\left(\bar{n}, \bar{p}, e^{+}, \bar{\nu}_{e}\right.$, and $\left.\bar{\chi}\right)$ are produced via the decays of $\phi$ particles in the processes

$\phi \rightarrow \chi+\nu+n \quad$ and then $n \rightarrow p+e^{-}+\bar{\nu}$ 
or

$\phi=\bar{\phi} \rightarrow \bar{\chi}+\bar{\nu}+\bar{n} \quad$ and then $\quad \bar{n} \rightarrow \bar{p}+e^{+}+\nu$.

In every separate decay (1) or (2), the baryon invariance is violated. If both decays (1) and (2) are equiprobable, then the system composed from matter, antimatter (including dark sectors), and a fraction of $\phi$-particles of the condensate, which have not decayed up to the instant under consideration, remains chargeless. The processes (1) and (2) imply that the particles and the antiparticles of dark matter are scalar $\left(\operatorname{spin} s_{\chi}=0\right)$ or vector $\left(s_{\chi}=1\right)$ particles. It follows from [3] that a dark matter particle may be a scalar particle with scalar interaction. The presence of a neutrino in decay (1) guarantees the spin conservation law.

The data of astrophysical observations indicate that matter dominates over antimatter in the observed part of the Universe, by pointing to the B-violation. According to the CPT-theorem, all interactions are invariant under the succession of the three operations $\mathrm{C}$, P, and $\mathrm{T}$ taken in any order. The baryon asymmetry of the Universe can be caused by the CP-violation. Supposing that this principle of quantum field theory also takes place for some unknown interaction, via which processes (1) and (2) occur, one can conclude that $\mathrm{CP}$ or $\mathrm{T}$-invariance breaks down in decays (1) and (2). The T-violation is confirmed by the fact that the probability of the inverse fusion reaction of three and more particles into one zero-momentum particle is negligibly small ${ }^{1}$. The $\mathrm{CP}$ non-invariance of this interaction supplies the arrow of time.

Particles and antiparticles in the decay products of processes (1) and (2) can annihilate between themselves and contribute to the cosmic background radiation. Net amounts of protons, leptons, and dark matter particles constitute matter in the luminous and dark forms in the Universe.

Taking the above-mentioned issues into account, let us examine decay (1), in which dark and baryonic matter are produced. We consider this process using an analogy with neutron and proton decays. In the standard model, the decay of a neutron is a result of the weak interaction mediated by the virtual $W$-boson exchange,

$n \rightarrow p+W^{-}$and then $W^{-} \rightarrow e^{-}+\bar{\nu}$.

\footnotetext{
1 This property is well known from the quantum scattering theory and the theory of chemical reactions, where it is shown that triple (and more multiple) simultaneous collisions are many orders of magnitude less probable than double collisions.
}

The decay rate $\Gamma_{n}$ of a neutron into the final $p e \nu$-state is equal to

$\Gamma_{n}=\frac{1}{\tau_{n}}=\alpha_{W}^{2} \frac{\Delta m^{5}}{M_{W}^{4}}$

(in units $\hbar=c=1$ ), where $\tau_{n}$ is the mean lifetime of a neutron, $\Delta m=m_{n}-m_{p}$ is the energy imparted by a $W$ boson to the leptons $e \nu, M_{W}$ is the mass of a $W$-boson, $m_{n}$ and $m_{p}$ are the masses of a neutron and a proton, respectively. The quantity $\alpha_{W}^{2}$ is a dimensionless constant, which characterizes the weak interaction strength and includes small radiative and other quantum corrections. This number is close to the value $G_{F} m_{p}^{2}=1.027 \times 10^{-5}$, where $G_{F}=1.166 \times 10^{-5} \mathrm{GeV}^{-2}$ is the Fermi coupling constant,

$\alpha_{W}^{2}=G_{F} m_{p}^{2}(1-\delta) \sim 10^{-5}$,

where $\delta \sim O\left(10^{-1}\right)$ is a small correction.

In any grand unified theory (GUT) (see, e.g., review [4]) the proton lifetime $\tau_{p}$ is given by the relation

$\Gamma_{p}=\frac{1}{\tau_{p}}=\alpha_{G}^{2} \frac{m_{p}^{5}}{M_{G}^{4}}$,

where $\Gamma_{p}$ is the decay rate of a proton, and $\alpha_{G}$ and $M_{G}$ are two parameters. The universal gauge coupling $\alpha_{G}$ is defined at the grand unification scale $M_{G}$. The SUSY GUTs give the value $\alpha_{G} \sim \frac{1}{25}$ at $M_{G} \sim 3 \times 10^{16}$ $\mathrm{GeV}$. These parameters correspond to the proton lifetime equal to $\tau_{p} \sim 10^{37} \mathrm{yr}$. We can use the estimation

$\alpha_{G}^{2} \sim 10^{-3}$.

It can be compared with $\alpha_{W}^{2}$ from Eq. (5):

$\alpha_{W}^{2} \sim 10^{-5}<\alpha_{G}^{2} \sim 10^{-3}$.

This relation is useful for the estimation of the coupling constant of the interaction via which processes (1) and (2) occur.

Let us suppose that the decay of a $\phi$-particle in process (1) is mediated by the exchange of virtual $X$-particles, which are quanta of some new field with the mass scale

$M_{X} \gg m_{\chi}>m_{n} \gg m_{\nu}$

where $m_{\chi}$ and $m_{\nu}$ are the masses of a dark matter particle and a neutrino. Generally speaking, a few versions of such a decay are possible:

$$
\begin{array}{cll}
\phi \rightarrow \chi+X & \text { and then } & X \rightarrow n+\nu, \\
\phi \rightarrow n+X & \text { and then } & X \rightarrow \chi+\nu \\
\phi \rightarrow \nu+X & \text { and then } & X \rightarrow \chi+n
\end{array}
$$


In Fig. 1, the diagram represents the first process in Eq. (10). The two other diagrams can be obtained by cyclic permutation of the particles $\chi, n$, and $\nu$.

Since the spin $s_{\phi}=0$, one concludes that, in the first process in Eq. (10), the spins of $\chi$ - and $X$-particles can vanish, $s_{\chi}=0$ and $s_{X}=0$, or be equal to $1, s_{\chi}=1$ and $s_{X}=1$. In this case, $\chi$ - and $X$-particles are scalars or vector bosons. In the second and third processes in Eq. (10), a $\chi$-particle is still a scalar or vector boson, but an $X$-particle is a fermion with the spin $s_{X}=\frac{1}{2}$.

We consider processes (10) by analogy to those described by Eqs. (4) and (6). We neglect the contribution from the integration with respect to the intermediate momentum of the $X$-particle in corresponding transition amplitudes, as well as the contributions to the decay rate of a $\phi$-particle $\Gamma_{\phi}$ arising from all higher-order diagrams. Then $\Gamma_{\phi}$ can be written as

$\Gamma_{\phi}=\alpha_{X}^{2} \frac{Q^{5}}{M_{X}^{4}}$,

where

$Q=m_{\phi}-\left(m_{\chi}+m_{n}+m_{\nu}\right)$

is the energy imparted by a $\phi$-particle at rest to the decay products in (1). The dimensionless coupling constant $\alpha_{X}^{2}$ takes the contributions into $\Gamma_{\phi}$ from the first or both the second and third pole diagrams for processes (10) into account. In the case where an $X$-boson has a supersymmetric partner with the same mass ( $X$-fermion), all three pole diagrams contribute to the decay rate $\Gamma_{\phi}$.

Using Eqs. (4), (6), and (11), one can write the expressions for the ratios of the decay rate of a $\phi$-particle to the neutron and proton decay rates:

$\frac{\Gamma_{\phi}}{\Gamma_{n}}=\frac{\alpha_{X}^{2}}{\alpha_{W}^{2}}\left(\frac{Q}{\Delta m}\right)^{5}\left(\frac{M_{W}}{M_{X}}\right)^{4}$

and

$\frac{\Gamma_{\phi}}{\Gamma_{p}}=\frac{\alpha_{X}^{2}}{\alpha_{G}^{2}}\left(\frac{Q}{m_{p}}\right)^{5}\left(\frac{M_{G}}{M_{X}}\right)^{4}$.

Here, the quantities $\Gamma_{\phi}, \alpha_{X}^{2}, Q$ and $M_{X}$ are unknown. The decay rate $\Gamma_{\phi}$ and the parameter $Q$ can be calculated independently in the kinetic theory which considers the two-step processes (1) and (2) as dynamical ones and uses the astrophysical data on the contributions of baryon, dark matter, and dark energy densities to the total matter-energy density budget in our Universe [5].

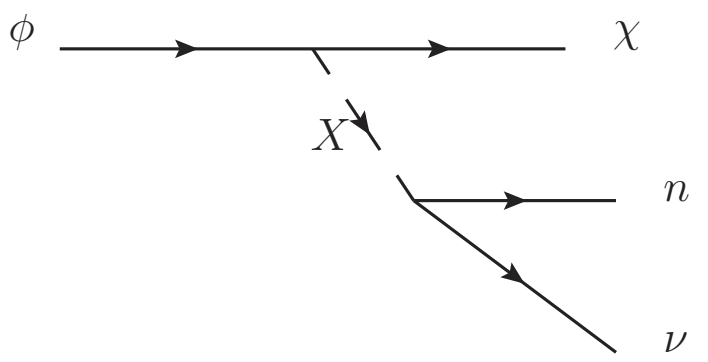

Fig. 1. Diagram which corresponds to the first decay in (10)

\section{Estimations of Coupling Constant and Mass Scale}

The Hubble expansion rate $H$ is a characteristic value, with which the decay rate $\Gamma_{\phi}$ can be compared. If $\Gamma_{\phi} \ll H$, baryons (and ordinary matter) will not be produced in the expanding Universe in this case. In another limiting case where $\Gamma_{\phi} \gg H$, the expansion of the Universe will not affect the decay rate $\Gamma_{\phi}$. This means that, for estimation, one can assume that the decay rate of a $\phi$-particle is close to the Hubble expansion rate. As a result, for the present-day Universe, we have

$\Gamma_{\phi} \approx 1.51 \times 10^{-42} \mathrm{GeV}$.

This value may be considered as the mean decay rate during the time interval $\Delta t=t_{0}$, where $t_{0}$ is the age of the Universe. Taking $\Gamma_{p} \approx 1.43 \times 10^{-69} \mathrm{GeV}$ and $\Gamma_{n}=0.74 \times 10^{-27} \mathrm{GeV}$, for the present epoch, we find

$\frac{\Gamma_{\phi}}{\Gamma_{p}} \sim 10^{27}, \quad \frac{\Gamma_{\phi}}{\Gamma_{n}} \sim 2 \times 10^{-15}$.

It is of interest that the ratio

$\frac{\Gamma_{n}}{\Gamma_{p}} \sim 5 \times 10^{41}$

gives the value, which is of the same order of magnitude as the well-known Eddington's magic numbers. It can be used for a more precise definition of the proton lifetime.

Equation (11) yields a relation between the mass scale $M_{X}$ and the imparted energy $Q$ at fixed values of $\Gamma_{\phi}$ and $\alpha_{X}$. The coupling constant $\alpha_{X}$ must satisfy the inequality

$\alpha_{W}^{2} \sim 10^{-5}<\alpha_{X}^{2}<\alpha_{G}^{2} \sim 10^{-3}$.

The energy $Q$ depends on the values of masses of $\phi$ - and $\chi$-particles. In Fig. 2, we show the mass of an $X$-particle as a function of $Q$.

For the coupling constant in interval (18), the mass scale $M_{X}$ takes the values

$0.2<\left(\frac{M_{X} \times 10^{-10}}{Q^{5 / 4}}\right)<0.5$. 


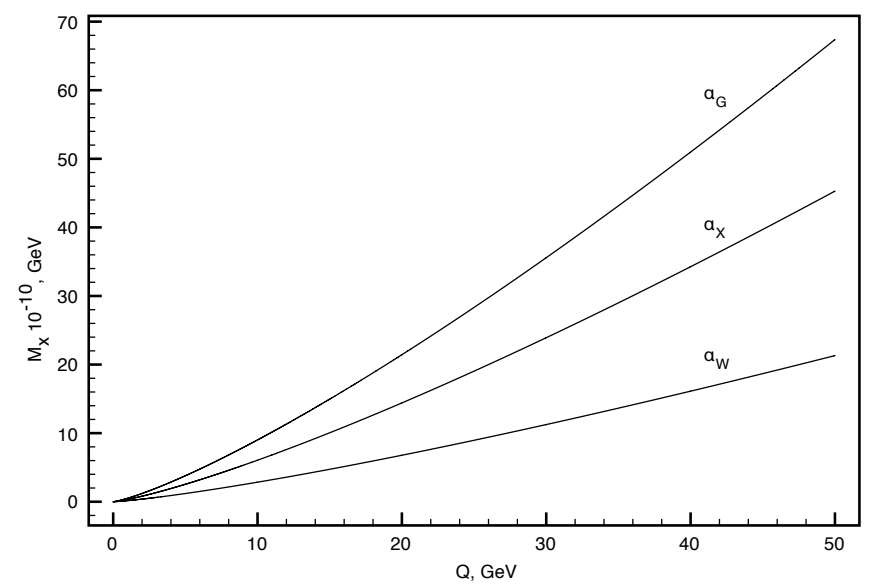

Fig. 2. Dependence of the $X$-particle mass $M_{X}$ on the energy $Q$ imparted by a $\phi$-particle to the decay products in process (1). The upper curve corresponds to the value $\alpha_{X}^{2}=\alpha_{G}^{2}=10^{-3}$; for the bottom curve, $\alpha_{X}^{2}=\alpha_{W}^{2}=10^{-5}$; the middle curve is drawn for the value $\alpha_{X}=1 / 70$

Reaction (1) with the production of baryons is possible, when the temperature of the primordial plasma in the early Universe is $T \lesssim 1 \mathrm{GeV}$ (which corresponds to the time $\left.t \gtrsim 10^{-6} \mathrm{~s}\right)^{2}$. Since $3-5$ particles are produced in the reaction, the imparted energy is $Q \lesssim 10 \mathrm{GeV}$. For $Q \sim 10 m_{p}$, the mass scale $M_{X}$ lies in the interval

$0.3<\left(M_{X} \times 10^{-11} m_{p}^{-1}\right)<0.9$,

and we can accept the numerical values

$\alpha_{X} \sim \frac{1}{70} \quad$ at $\quad M_{X} \sim 6 \times 10^{10} \mathrm{GeV}$

for the parameters of the interaction which causes decay (1). The radius of action of the corresponding force is

$R_{X}=M_{X}^{-1} \sim 3 \times 10^{-25} \mathrm{~cm}$.

This gives the value

$n_{\phi} \sim M_{X}^{3} \sim 10^{73} \mathrm{~cm}^{-3}$

for the density of dark energy quasiparticles considered as $\phi$-particles surrounded by the cloud of virtual $X$ particles. The $\phi$-particle is massive, and it can, in principle, exhibit itself through the gravitational action, but its gravitational coupling constant is expected to be very small [5]. The coupling constant $\alpha_{X} \sim 10^{-2}$ has the same order of magnitude as the fine structure constant $\alpha \approx \frac{1}{137}$. This allows us to consider the subtle processes,

\footnotetext{
2 The higher temperatures correspond to the era of the quarkgluon plasma in the early Universe.
}

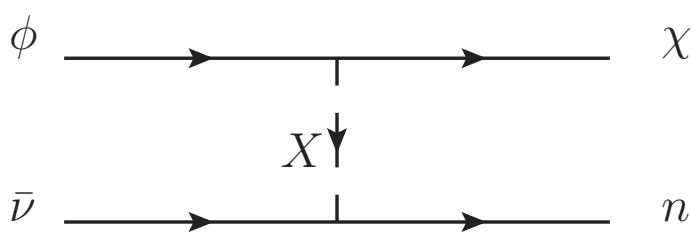

Fig. 3. Diagram describing the reaction (24)

which occur via the virtual $X$-particle exchange as more realistic for the identification of dark matter and dark energy.

Let us consider the reaction

$\bar{\nu}+\phi \rightarrow \chi+n$,

in which dark matter particles are produced. In Fig. 3, the diagram of this reaction is shown.

The corresponding cross-section is equal to

$\sigma(\bar{\nu} \phi) \simeq \frac{\Gamma_{\phi}}{v M_{X}^{3}}$

where $v$ is the relative $\bar{\nu}-\phi$ motion velocity, and the denominator is the incident flow of antineutrinos in the rest frame of a $\phi$-particle. Since, in this reference frame, the velocity $v$ is equal to the velocity of the incident antineutrino, we can take $v \approx c$. For the decay rate (15) and the mass scale $M_{X}$ from (21), we obtain

$\sigma(\bar{\nu} \phi) \sim 0.7 \times 10^{-74} \mathrm{GeV}^{-2}$.

Dark matter particles can be produced in another reaction as well:

$\bar{n}+\phi \rightarrow \chi+\nu$

For nonrelativistic antineutrons, the cross-section of this process is

$\sigma(\bar{n} \phi) \simeq \frac{0.7}{\sqrt{E_{\bar{n}}}} \frac{\Gamma_{\phi}}{M_{X}^{3}}$,

where $E_{\bar{n}}$ is the kinetic energy of an antineutron taken in $\mathrm{GeV}$. For thermal and fast antineutrons, the crosssections are equal to

$\sigma(\bar{n} \phi) \sim 10^{-69} \mathrm{GeV}^{-2}$ for $E_{\bar{n}}=0.25 \times 10^{-10} \mathrm{GeV}$,

$\sigma(\bar{n} \phi) \sim 1.6 \times 10^{-73} \mathrm{GeV}^{-2}$ for $E_{\bar{n}}=10^{-3} \mathrm{GeV}$,

respectively. These values are much less than the elastic scattering cross-section of a dark matter particle with a nucleus $\sigma \sim 10^{-13} \mathrm{GeV}^{-2}$ used for the estimation of a dark matter particle mass $[3,6]$. 


\section{Concluding Remarks}

In the early Universe, the imparted energy $Q$ has to be proportional to the hot plasma temperature, $Q \sim T$. From (11), it follows that the rate $\Gamma_{\phi} \sim T^{5}$ at high temperatures. During the expansion of the Universe, the temperature decreases, the rate $\Gamma_{\phi}$ decreases as well, and it tends to the mean value (15) at low temperatures $(t \gg 1 \mathrm{~s})$.

The density of dark energy particles (23) calculated at $Q \sim 10 m_{p}$ shows that, after the space averaging, the volume $\sim(2 \mathrm{~m})^{3}$ contains the same number of dark energy particles as the number of equivalent baryons $\sim 10^{80}$ in the observed part of our Universe. The cross-sections of reactions (24) and (27) via the virtual $X$-particle exchange are very small.

Within the framework of the quark model of baryons, one may conclude that the condensate (and, hence, dark energy) can be a chargeless aggregate of pointlike quarks and gluons, a kind of quark-gluon plasma. Then $\phi$-particles will be the particle-like excitations of this plasma. The existence of virtual $X$-particles gives the possibility for $\phi$-particles to decay with a very small probability into the observed luminous and dark matter. As regards an $X$-particle, it can be one of the supersymmetric particles.

Another point of view might be possible: the quarkgluon plasma is formed after the disintegration of baryons into quarks and gluons at high temperatures in the early Universe, whereas baryons themselves appear as a result of the decay of $\phi$-particles of the condensate. But such a model does not give an answer to the question about the nature of the condensate (dark energy).

Using the hypothesis about the existence of a new scale $M_{X} \sim 10^{10} \mathrm{GeV}$, a more general scheme of the unification of now four (without gravitation) fundamental interactions into one single force with a scale $M_{G} \sim 10^{16}$ $\mathrm{GeV}$ may be proposed in the framework of the quark model of baryons. In the simplest version of a new theory, some new gauge group $G^{\prime}$ must be included formally into the scheme

$G \stackrel{G}{\longrightarrow} G^{\prime} \stackrel{X}{\longrightarrow} S U(3) \times S U(2) \times U(1) \stackrel{W}{\longrightarrow} S U(3) \times U(1)$,

where $G$ is some group, which realizes the local symmetry principle and contains the group $G^{\prime}$ as a subgroup. The model with such a group $G$ must have three mass scales: the masses of gauge particles $G, X$, and $W$. The values of these masses $M_{G}, M_{X}$, and $M_{W}$ characterize the spontaneous symmetry breaking of $G$ to $G^{\prime}$, then to $S U(3) \times S U(2) \times U(1)$, and finally to $S U(3) \times U(1)$. Since $M_{G} \gg M_{X} \gg M_{W}$, there is a vast hierarchy of gauge symmetries. In order to build the grand unification theory, which will realize scheme (30), it is necessary to make the group $G$ specific.

1. E.W. Kolb and M.S. Turner, The Early Universe (Addison-Wesley, Redwood City, 1990).

2. V.E. Kuzmichev and V.V. Kuzmichev, Acta Phys. Pol. B 39, 2003 (2008), arXiv:0712.0465 [gr-qc]; ibid. 39, 979 (2008), arXiv:0712.0464 [gr-qc]; ibid. 40, 2877 (2009), arXiv:0905.4142 [gr-qc]; Ukr. J. Phys. 55, 626 (2010).

3. A.L. Fitzpatrick, D. Hooper, and K.M. Zurek, arXiv:1003.0014 [hep-ph] (2010).

4. K. Nakamura et al., (Particle Data Group), J. Phys. G 37, 075021 (2010).

5. V.E. Kuzmichev and V.V. Kuzmichev, arXiv:1011.6196 [hep-ph] (2010).

6. C.E. Aalseth et al. (CoGeNT), arXiv:1002.4703 [astroph.CO] (2010).

Received 15.07.11

\section{ЩОДО УТВОРЕННЯ МАТЕРIї У ВСЕСВITI}

\section{В.Є. Кузъмичов, В.В. Кузъмичов}

$\mathrm{P}$ е $з$ ю м е

Запропоновано модель утворення звичайної та темної матерії у розпаді гіпотетичного антигравітаційного середовища у формі конденсату безспінових масивних частинок. Розпади цих частинок на баріони, лептони та частинки темної матерії зумовлені дією певної взаємодії з масштабом маси між електрослабкою взаємодією та великим об'єднанням. Темну енергію, що спостерігається, можна ототожнити з частиною конденсату, яка не розпалась до моменту вимірювання. Імовірність розпаду частинок конденсату виражається через три параметра константу зв'язку $\alpha_{X}$, масштаб маси $M_{X}$, що визначає масу $X$-частинки як посередника взаємодії, та енергію, яка передається продуктам розпаду. У припущенні, що константа розпаду частинок конденсату є величиною того самого порядку, що і стала Хаббла, отримано обмеження на можливі значення маси $M_{X}$. За характерне значення константи зв'язку можна прийняти $\alpha_{X} \sim \frac{1}{70}$ при $M_{X} \sim 6 \cdot 10^{10}$ ГеВ. Розраховано перерізи реакцій, в яких можуть утворюватись частинки темної матерї. 